simultaneous hybridization of multiple probes our method employs successive probe hybridization, amplification, and development of substrate signal for each RNA target molecule, followed by removal of probe and associated DNA branches by a highstringency wash. Steps are illustrated in figure 1. A single-plex, and a standard dapB negative control was run in parallel for each probe.

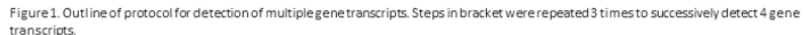
transcripts.

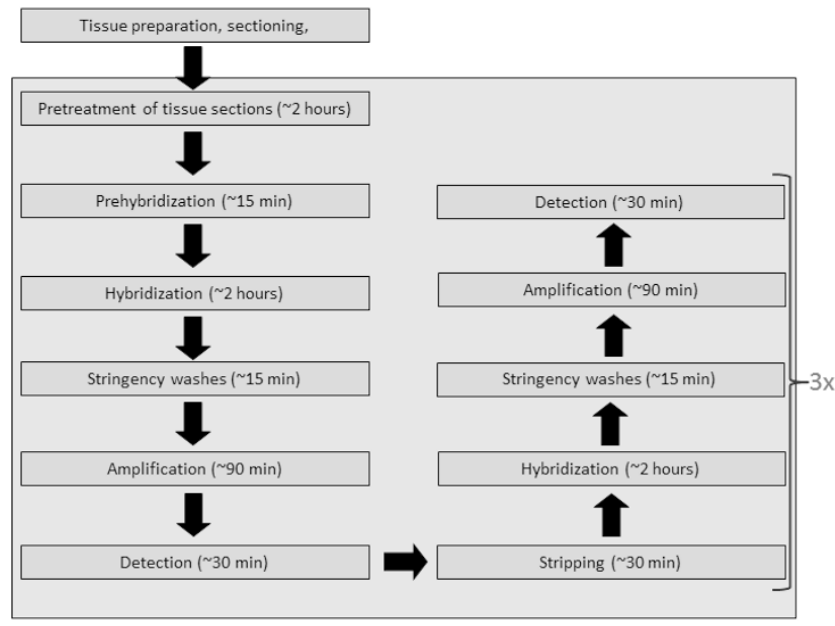

Results: Data are presented for co-expression of interneuron markers in adult mouse brain in figure 2. Formalin-fixed, paraffin-embedded coronal sections from adult mouse brain were used for successive hybridization with four different gene-specific probe sets for 4 interneuron markers: Somatostatin (Sst), Parvalbumin (Pvalb), Vasoactive intestinal polypeptide (Vip), Neuropeptide Y (Npy).
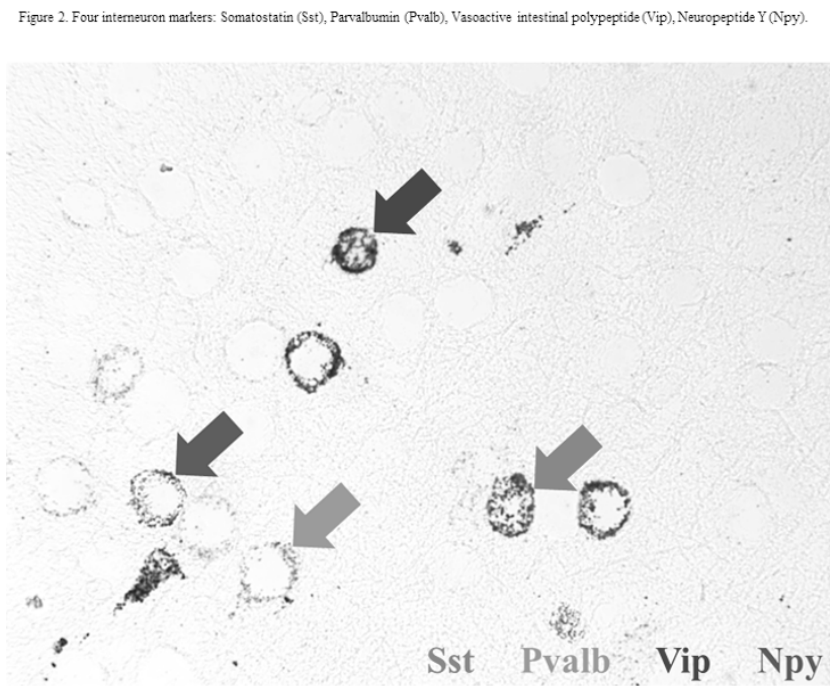

Conclusions: Sequential ISH using gene-specific oligonucleotide probes with branched DNA signal amplification allows for sensitive and specific detection of multiple distinct RNA target transcripts in the same tissue section without a decrease in the sensitivity and specificity of the assay. This method is valuable not only for the co-localization of marker expression but also in circumstances when tissue resources are limited.

2178 A Novel Computerized Image Analysis Method to Differentiate Malignant Mesothelioma from Reactive Mesothelial Proliferations in Effusion Cytology Specimens

$O$ Yergiyev, AB Tosun, GK Rohde, JF Silverman. Allegheny General Hospital, Pittsburgh, PA; Carnegie Mellon University, Pittsburgh, PA; West Penn Hospital, Pittsburgh, PA. Background: Differentiating malignant mesothelioma (MM) from reactive mesothelial proliferations (RMP) is based on demonstrating tissue invasion. While pleural effusion specimens can yield cells for cytologic examination, it is insufficient to make the definitive diagnosis of MM since tissue invasion cannot be assessed. We evaluated a novel image analysis technique which can potentially differentiate MM from RMP based on the patterns of chromatin distribution in the nuclei of mesothelial cells in effusion cytology specimens.

Design: 34 cases were selected from the pathology database of two tertiary care academic centers, including 16 cases of MM and 18 cases of RMP. All patients had both a pleural effusion cytology specimen and pleural biopsy with histologic confirmation. Diff-Quik stained slides from each case were reviewed, and digital images of mesothelial cells were acquired using standard imaging equipment. 1080 individual nuclei were segmented using original software. After drawing the nuclear contours, the luminance component of segmented nuclei was analyzed. The images were first normalized to remove relative translations and rotations. After normalization, the chromatin content of each segmented nucleus was processed to obtain its linear optimal transport (LOT) embedding. A linear classification function was computed for each individual nucleus to distinguish it from the two classes (benign and malignant) by using the modified Fisher Linear Discriminant Analysis (PLDA) technique on the LOT embedding. A set of nuclei from each patient was then classified using a majority voting strategy. Results: We utilized the standard "leave one patient out" cross validation strategy to classify the cases. Each patient was diagnosed by using the above classifier, computed without using nuclei from that patient. In each classification task the same number of nuclei for each class was used. With the above technique, we were able to classify the effusion cytology cases as benign or malignant with $100 \%$ accuracy when compared to the histologic diagnosis.

\begin{tabular}{||l|l|l||}
\hline \hline & Predicted \\
\hline \hline Actual & Benign & Malignant \\
\hline Benign & 18 & 0 \\
\hline Malignant & 0 \\
\hline Accuracy & $100 \%$ & 16 \\
\hline
\end{tabular}

Accuracy

Table 1 Study results.

Conclusions: We present an original computerized technique with a potential to differentiate between MM and RMP based on the nuclear structure of mesothelial cells in effusion cytology specimens, that may alleviate the need for an invasive procedure.

\section{Ultrastructural Pathology}

2179 Three Year Follow Up in an Allograft Kidney from a Metachromatic Leukodystrophy Donor - Ultrastructural Findings

$S$ Ballal, $N$ Sondheimer, S Koutzaki, A Doyle, S Soundararajan. Drexel University College of Medicine, Philadelphia, PA; The Children's Hospital of Philadelphia, Philadelphia, PA.

Background: Metachromatic leukodystrophy(MLD) is an inherited disorder of myelin metabolism associated with neurodegenerative changes and deposition of metachromatic sulfatide granules in the nervous system. Viscera with excretory functions are also affected. Ultrastructural studies of the kidney in MLD reveal lamellar or zebroid inclusions bound by a single unit membrane. Organs of these patients have rarely been used for donation.

Design: We present the ultrastructural findings over a three year period of a successfully transplanted kidney from a 10 year old MLD donor, diagnosed at age 2 who suffered cardiac arrest. His kidney was transplanted into a 61 year old woman with diabetic nephropathy. Post-transplant course was uncomplicated, with immediate graft function. Currently, the patient is well with creatinine of $1.1 \mathrm{mg} / \mathrm{dl}$.

Results: Annual surveillance biopsies revealed acute cellular rejection at 1 year and mild patchy interstitial fibrosis and tubular atrophy at 3 years. Electron microscopic examination revealed intra-cytoplasmic membrane bound concentrically arranged dense laminated inclusions ranging from $1-2 \mu \mathrm{m}$ in the proximal and distal tubules.

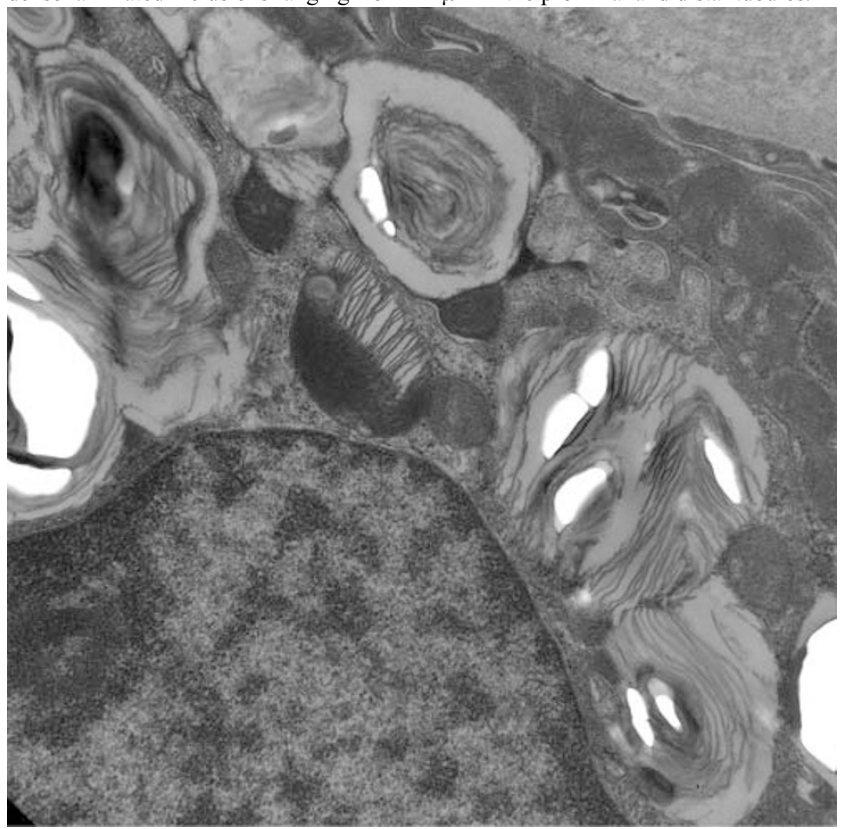

At 3 years, the inclusions are intralysosomal, fragmented and dispersed within the cytoplasm of the affected tubules. There is focal disruption of the brush border through which they are extruded. 


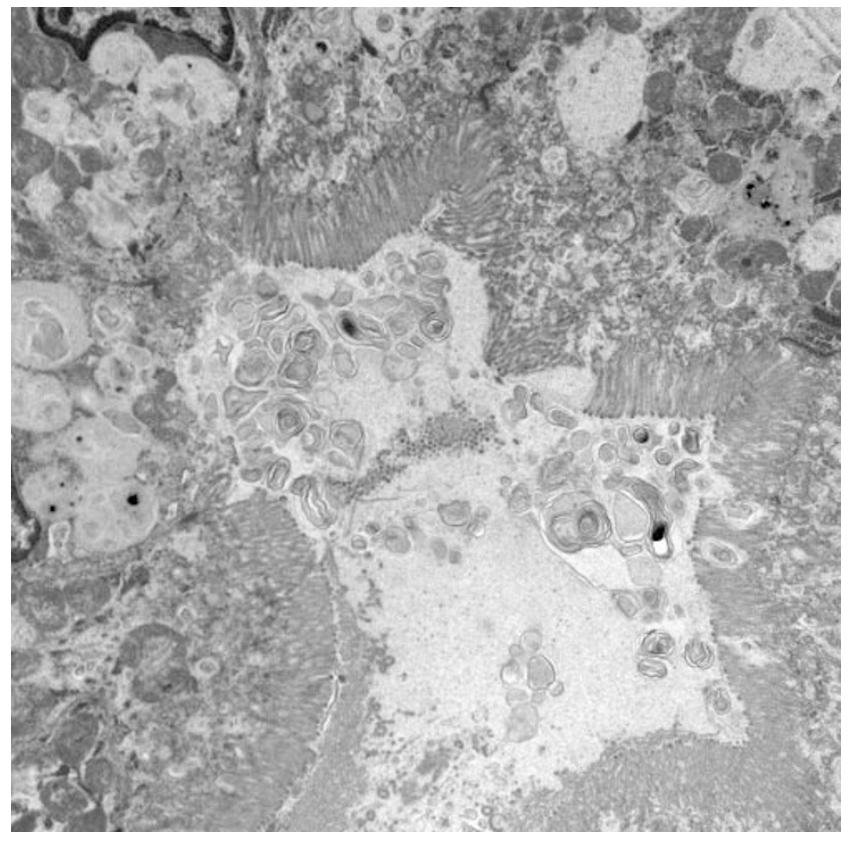

No glomerular or vascular morphological abnormalities are identified.

Conclusions: Eligible MLD donor kidneys reveal persistent intracytoplasmic zebroid inclusions in the proximal and distal tubules with evidence of lysosomal digestion and excretion into the urinary space. These inclusions do not adversely affect the allograft function.

\section{Ultrastructural Characterization of Papillary Renal Cell} Carcinoma and Oncocytoma

RM Bhatti, KA La Fortune, YD Mo, CP Helen. University of Virginia, Charlottesville, VA.

Background: A small proportion of renal tumors are difficult to classify using light microscopy, and immunohistochemistry is often unhelpful in these cases. Renal tumors with oncocytic features are particularly problematic. Electron microscopy (EM) is an ancilliary tool that has been used to help categorize challenging renal tumors. The ultrastructural features of papillary renal cell carcinoma (PRCC) have been poorly characterized to date. We therefore examined Type 1 and Type 2 PRCC, including oncocytic and sarcomatoid variants, and compared them with oncocytomas.

Design: Eleven renal tumors, diagnosed from 2007-2013, were selected for ultrastructural analysis. One formalin-fixed case was retroprocessed. Review of light microscopy revealed 9 cases of PRCC, (four Type 1 and five Type 2), and two oncocytomas. One of the Type 1 and two of the Type 2 PRCC had oncocytic features, defined as having copious granular eosinophilic cytoplasm. Two of the Type 2 PRCC had sarcomatoid areas. Mitochondrial density was defined as rare when mitochondria occupied $\leq 5 \%$ of the cytoplasm, few $6-25 \%$, moderate $26-75 \%$ and numerous $76-100 \%$. Results: Among PRCC, mitochondria completely filled the cytoplasm in 1/4 (25\%) Type 1 cases and in $3 / 5(60 \%)$ Type 2 cases.

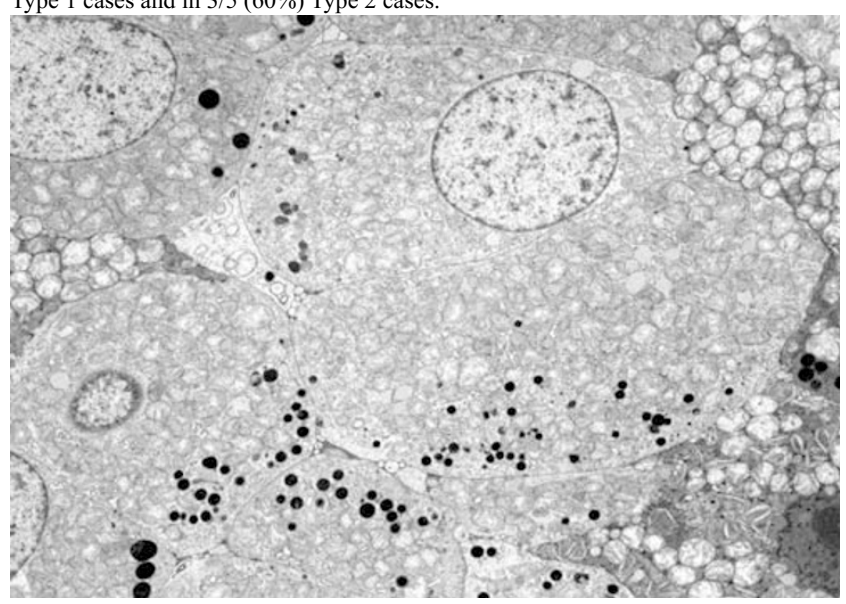

In the remaining Type 1 PRCC, mitochondria were moderate in number, but were also diffusely distributed. The density of mitochondria correlated poorly with oncocytic features as evaluated by light microscopy. One sarcomatoid Type 2 case had only rare mitochondria towards the luminal pole, while the other case had moderate numbers of diffusely distributed mitochondria. Both oncocytomas had mitochondria of similar size occupying the entire cytoplasm. The mitochondrial cristae were fractured and variably shortened in both types of PRCC, whereas they were longer and more numerous in oncocytomas.
Conclusions: Type 1 and Type 2 PRCC cannot be reliably distinguished based upon ultrastructural features. The density of mitochondria does not correlate with the oncocytic features of PRCC as evaluated by light microscopy. Furthermore, ultrastructural analysis is unlikely to help distinguish between the PRCC and oncocytomas, because of overlapping mitochondrial features. EM may however, be used to refine the controversial oncocytic variant of PRCC.

2181 Amyloidogenesis in Mesangial Cell: Lysosomal Processing to Fibril Formation and Delivery to the Extracellular Matrix (ECM)

GA Herrera, J Teng, EA Turbat-Herrera. Louisiana State University Health Sciences Center, Shreveport, LA.

Background: The process of amyloid formation in mesangial cells requires phenotypic transformation from a smooth muscle to a macrophage phenotype and endocytosis of glomerulopathic light chains (GLCs) with eventual delivery to the mature lysosomal system. Fibril formation in lysosomes and export of fibrillary material to the extracellular domain (ECM) represent crucial events in the process that have not been fully characterized.

Design: MCs incubated with amyloidogenic light chains $(\mathrm{N}=15)$ and glomeruli from mice injected via penile vessels with amyloid-forming light chains $(n=8)$, results in amyloid formation, the latter in the mesangium.

Results: The samples obtained in different time frames. (2, 4,6,12 hours and 2,4,6,8 days) post initial treatment was examined by light, transmission and scanning electron microscopes. Focusing on lysosomes in MCs, as they processed LCs and formed fibrils, Scanning EM was very useful in depicting surface events in MCs associated with amyloid fibril extrusion into the ECM.

Conclusions: Amyloid fibril formation in lysosomes in MCs is clearly identified. Lysosomes then abut and fuse with the MC membranes where gaps are identified and expel their fibrillary contains into the ECM. These events are also clearly depicted in the experimental platform exanimated.

2182 Cholesterol Ester Storage Disease: Role of Electron Microscopy in Diagnosis and Guiding Molecular Confirmation

$J$ Hicks, E Wartchow, G Mierau. Texas Children's Hospital, Houston, TX; Children's Hospital Colorado, Aurora, CO.

Background: Cholesteryl ester storage disease (later-onset CESD; infantile-onset Wolman disease) is caused by deficient lysosomal acid lipase activity, resulting in cholesteryl ester accumulation in the liver, spleen and macrophages throughout the body. Lipase A (LIPA) gene mutation (10q23.2-q23.3) is responsible for this autosomal recessive disease that can present during the $1^{\text {st }}$ year of life and into adulthood. This storage disease is characterized by microvesicular steatosis leading to liver failure, accelerated atherosclerosis and premature demise. Although CESD is rare, many affected patients are unrecognized or misdiagnosed as nonalcoholic steatohepatitis, non alcoholic fatty liver disease or cryptogenic liver disease.

Design: The surgical pathology archive of two pediatric hospitals identified 10 children (age range 4 months to 10 years, 5 females, 5 males) with steatotic liver disease and liver biopsy tissue available for evaluation of CESD by electron microscopy. All neonates, infants and children presented with elevated liver enzymes, liver dysfunction and mild to moderate hepatomegaly. Common symptoms were vomiting, diarrhea, growth retardation, failure to thrive, abdominal distention, and malabsorption.

Results: All liver biopsies on routine histopathologic examination demonstrated diffuse microvesicular steatosis without minimal zonal differences in the lobules. There were also markedly vacuolated histiocytes within the portal tracts and areas with fibrous tissue, as well as vacuolated Kupffer cells. Electron microscopic examination demonstrated numerous membrane-bound lipid vacuoles filling and distending the cytoplasm of the hepatocytes. In addition, there were variable numbers of needle-shaped cholesterol ester crystals in the cytoplasm of the hepatocytes.

Conclusions: Microvesicular steatosis may have many different etiologies, including mitochondrial diseases, mucolipidoses, medication effects, obesity, glycogen storage disease, cystic fibrosis, Reye syndrome, medium and long-chain fatty acid disorders, fatty acid oxidation disorders and CESD/Wolman disease. The finding of diffuse membrane-bound lipid vacuoles with needle-shaped cholesterol ester crystals provide definitive evidence for cholesterol ester storage disease. This electron microscopic feature guides appropriate lysosomal acid lipase activity evaluation and targeted genetic testing for LIPA gene mutational analysis for autosomal recessive CESD in the affected neonate and family members.

2183 Alveolar Soft Part Sarcoma: Role of Electron Microscopy in Diagnosis and Directing Molecular Genetic Evaluation

$J$ Hicks, E Wartchow, G Mierau. Texas Children's Hospital, Houston, TX; Children's Hospital Colorado, Aurora, CO.

Background: Alveolar soft part sarcoma (ASPS) is a rare tumor first described in 1952. It accounts for $0.5-1 \%$ of soft tissue sarcomas and affects mainly adolescents and young adults. The name "alveolar" was derived from its pseudoalveolar appearance with clustered polygonal cells lacking central cohesion. Tumor cells exhibit characteristic PAS-positive, diastase-resistant intracytoplasmic rhomboid crystals that contain monocarboxylate transporter 1 and CD147. ASPS possesses the chromosome rearrangement $\operatorname{der}(17) \mathrm{t}(\mathrm{X} ; 17)(\mathrm{p} 11 ; \mathrm{q} 25)$ resulting in ASPL-TFE3 gene fusion.

Design: Surgical pathology archives of two pediatric hospitals identified 10 cases of soft tissue sarcomas consistent with ASPS with tissue available for electron microscopy. Study population consisted of 6 females and 4 males with an age range of 5 to $19 \mathrm{yrs}$ (12-19 yrs of age ([=9]; one $5 \mathrm{yr}$-old). Asymptomatic masses presented in the tongue $(3 / 10)$, abdominal wall $(2 / 10)$, forearm $(2 / 10)$, thigh $(1 / 10)$, submandibular area $(1 / 10)$, and pharynx (1/10). TFE3 immunostaining was available for review in 7 cases. 
Results: Histopathologic examination showed variably-sized nests of round to polygonal tumor cells surrounded by sinusoidal vessels within connective tissue. TFE3 immunostaining showed nuclear positivity with tumor cells (7/7). Some tumor cells had vague rhomboid to rod-shaped cytoplasmic inclusions (PAS-diastase in 4 cases). Electron microscopy showed discontinuous basal lamina, poorly developed cell junctions, frequent mitochondria, rough endoplasmic reticulum and Golgi complexes. The hallmark ultrastructural feature was membrane-bound rhomboid to rectangular to rod-shaped crystalline structures. Crystalline structures had a well-delineated lattice pattern comprised of rigid fibrils $(5-7 \mathrm{~nm})$ with a $10-12 \mathrm{~nm}$ periodicity.

Conclusions: The characteristic crystalline structures by electron microscopy confirm the diagnosis of ASPS, and eliminate entities such as metastatic conventional and clear cell renal cell carcinoma, granular cell tumor (TFE3 positivity in certain proportion of tumors), myelomelanocytic tumors, paraganglioma, rhabdomyoma, hibernoma, and clear cell sarcoma of soft tissues from consideration. Further confirmation may be attained by identification of the $\operatorname{der}(17) \mathrm{t}(\mathrm{X} ; 17)(\mathrm{p} 11 ; \mathrm{q} 25)$ with $A S P L-T F E$ fusion. Accurate diagnosis of ASPS is important because of resistant to conventional cytotoxic chemotherapy and need for complete tumor excision. Recently, promising results with antiangiogenic agents (bevacizumab, topotecan, sunitinib malate, cediranib) have been reported.

\section{Microvillus Inclusion Disease: Role of Electron Microscopy in Guiding Molecular Diagnosis}

J Hicks, E Wartchow, G Mierau. Texas Children's Hospital, Houston, TX; Children's Hospital Colorado, Aurora, CO.

Background: Microvillus inclusion disease (MVID) is a congenital defect in enterocytes resulting in attenuation or loss of surface microvilli and presence of cytoplasmic microvillus inclusions. This autosomal recessive condition is characterized by persistent neonatal secretory diarrhea and the inability to absorb nutrients from the intestinal lumen. $M Y O 5 B$ gene mutation that encodes for the ubiquitously expressed myosin $\mathrm{Vb}$ protein is responsible for this condition. The differential diagnosis for persistent neonatal diarrhea includes MVID, celiac disease, autoimmune enteropathy, tufting enteropathy, allergic enteropathy, cystic fibrosis, neonatal inflammatory bowel disorder, chloride or sodium diarrhea, $\mathrm{Na}-\mathrm{H}$-exchange deficiency, glucose-galactose, malabsorption, sucrase-isomaltase deficiency, and rarely intestinal pseudo-obstruction syndrome or motility disorders.

Design: The surgical pathology archives at 2 pediatric hospitals identified 12 neonates and infants with a clinical history of persistent diarrhea and with intestinal biopsies available for evaluation of MVID by electron microscopy. The study population consisted of 6 males and 6 females who underwent endoscopic small intestine and/ or colon biopsies for persistent neonatal/infantile diarrhea. Tissue was available for electron microscopic review and evaluation.

Results: With electron microscopic examination, there were extensive microvilli abnormalities seen with all biopsy specimens. The microvilli were markedly attenuated to absent. When present the microvilli appeared to be foreshortened, widened, clubbed, or fused. The cytoplasmic findings were variable with some enterocytes demonstrating typical microvillus inclusions, and others with moderate to large-sized vesicles in the apical cytoplasm. In some instances, variably-sized vesicles were seen diffusely throughout the cytoplasm. The vesicles often contained electron lucent material. There were also increased lysosomal-like bodies.

Conclusions: Although MVID diagnosis may be suspected on PAS, CD10, villin and CEA staining, electron microscopy provides definitive evidence for the diagnosis of MVID. Establishment of this diagnosis is important in eliminating other etiologies of persistent neonatal diarrhea. Electron microscopic findings guide appropriate molecular genetic evaluation for $M Y O 5 B$ gene mutational analysis for both the neonate and family members, and avoids unnecessary genetic testing for other entities associated with neonatal diarrhea.

2185 Gastric Light Chain Deposition in the Form of Immunotactoid Gastropathy

K-Y Jen, OK Fix, EN Foster, ZG Laszik, LD Ferrell. University of California San Francisco, San Francisco, CA; Kaiser Permanente, Vacaville, CA.

Background: Immunotactoid (IT) deposits are defined by their ultrastructural appearance and consist of microtubular structures typically measuring $>30 \mathrm{~nm}$ in diameter. Although a rare entity, IT deposition most often manifests as IT glomerulopathy and is associated with underlying lymphoplasmacytic disorders. IT deposition in other organs is exceedingly rare and has never been reported in the stomach.

Design: The ultrastructural findings of IT deposition in the stomach are presented. A 58-year-old female with cirrhosis and hepatocellular carcinoma (status post radiofrequency ablation) due to chronic hepatitis $\mathrm{C}$ virus infection underwent endoscopy for evaluation of varices. Examination of the stomach revealed punctate erosions in the antrum. Similar lesions were noted one year prior. The antral lesions were biopsied at both points in time.

Results: Microscopic examination of both gastric biopsies revealed abundant amorphous eosinophilic material expanding the lamina propria. This material was Congo Rednegative, but immunohistochemical staining for kappa light chain was diffusely and strongly positive, while lambda light chain showed weak focal staining. Electron microscopy disclosed widespread, abundant microtubular IT deposits infiltrating the lamina propria of the stomach and basement membranes of the gastric glands. The microtubules were readily recognizable at 5,000X magnification, showing straight and curvilinear configurations with areas of parallel arrangement. The deposits measured 62 $\mathrm{nm}$ in average diameter. Liquid chromatography tandem mass spectrometry confirmed that the deposits consisted of kappa light chains. The patient displayed no renal signs or symptoms to indicate presence of IT glomerulopathy. Additional thorough clinical evaluation failed to detect paraproteinemia or a lymphoplasmacytic disorder.
Conclusions: This case represents the first report of IT deposits in the stomach, which we term immunotactoid gastropathy. The deposits consist of Congo Red-negative kappa light chains with ultrastructural characteristics very similar, if not identical, to that seen in IT glomerulopathy. However, unlike patients with IT deposits in the kidney, this patient showed no evidence of paraproteinemia or lymphoplasmacytic disorder.
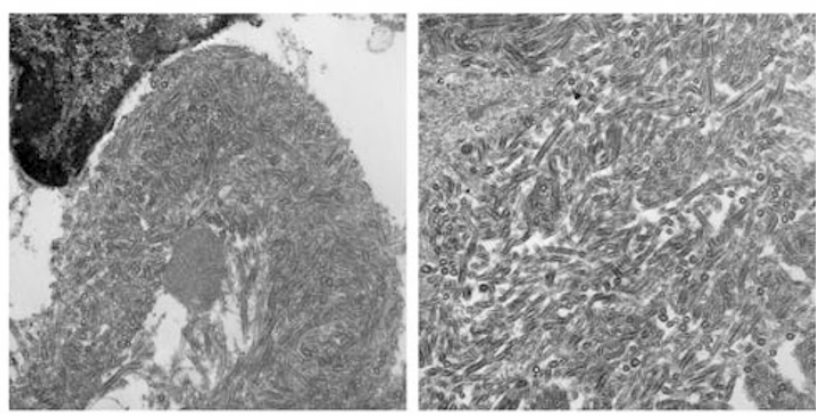

2186 Mammary Analogue Secretory Carcinoma of the Parotid Gland: A Comparative Ultrastructural Study

JA Merino, M Simon, N Juanpere, T Baro, JL Alvarez, JM Corominas, S Serrano, J Lloreta. Hospital del Mar, Barcelona, Spain; Pompeu Fabra University, Barcelona, Spain

Background: Mammary analogue secretory carcinoma of the parotid has been recognized recently as a locally aggressive tumor with a distinctive cytogenetic translocation $(\mathrm{t}(12 ; 14)(\mathrm{p} 17 \mathrm{q} 25)$. There are very few reports on this tumor and most of them have very little information on their ultrastructural features. The purpose of the present study has been to analyze the electron microscopic features of one of these cases and to compare it with the mammary counterpart.

Design: A left parotideal mass was found in a 35 year-old woman. The mass was surgically resected and samples for histology and for electron microscopy were taken. Serial sections were stained with antibodies for queratins (AE1-AE3, Cam 5.2, 34BetaE12, queratin 7), p63, EMA, CEA, smooth muscle actin, muscle specifcic actin, S100, vimentin, and GCDFP15. Tissue for electron microscopy was fixed in glutaraldehyde and osmium tetroxide, embedded in resin, thin-sectioned and examined with a FEI CM100 transmission electron microscope.

Results: By light microscopy, the tumor was well circumscribed and was composed of aggregates of cells with somewhat inconspicuous cell borders, with small vacuoles, lumina and microcystic spaces.By immunohistochemistry, moderate expression of Cam 5.2 and keratin 7, weak expression of EMA, S100, and vimentin, as well as negative p63, muscle specific and smooth muscle actins. were found. GCDFP15 was positive only in isolated cells. On ultrastructural examination, there were prominent invaginations of the cell surface studded with microvilli, and some intra- and intercellular lumina also with a microvillous surface. The cytoplasm contained abundant mitochondria surrounded by rough endoplasmic reticulum. Very isolated cells contained large granules that could be zymogen granules. The ultrastructural features of MASC would suggest a complex phenotype combining intercalated and striated duct traits with isolated true secretory cells with acinar differentiation. In contrast, mammary secretory carcinomas tend to have intercellular, but not so many intracellular lumina, as well as a more prominent secretory component.

Conclusions: The so-called mammary analogue secretory carcinoma of the salivary gland does not reproduce the ultrastructural features of secretory carcinoma of the breast. Electron microscopic findings suggest a predominantly proximal ductal phenotype (intercalated and striated ducts) with scanty zymogen secreting cells.

\section{Ciliary Inclusion Disease: Report of a New PCD Variant}

EP Wartchow, R Jaffe, G Mierau. Children's Hospital Colorado, Aurora, CO; University of Pittsburgh School of Medicine, Children's Hospital of UPMC, Pittsburgh, PA.

Background: Primary ciliary dyskinesia (PCD) is a rare, inherited disorder affecting cilia motility and thus impairing mucus clearance of the respiratory tract. With the exception of a single known mutation, all cilia dysfunction associated with PCD has been attributed to a variety of consistently observed structural abnormalities within individual cilia. While basal body migration abnormalities have been implicated in other ciliopathies, this mechanism has yet to be associated with congenital airway disease. Design: The study consists of 8 biopsies from 5 patients ( 4 male, 1 female), ages 1-5 years old, each suspected of having PCD. Clinical features of the patients included situs inversus, dextrocardia, obstructive rhinitis, recurrent pneumonia, tracheobronchomalacia and recurrent sinopulmonary infection. In 3 of the 5 patients, multiple biopsies were obtained and evaluated.

Results: Electron microscopic evaluation of each specimen showed that few cilia were present at the respiratory epithelial cell surface, however those that were observed displayed a normal ultrastructural appearance and axonemal architecture. Within many of the cells, including those deep within the epithelial cell layer, were large intracytoplasmic vesicular inclusions containing disorganized arrays of structurally normal cilia. 


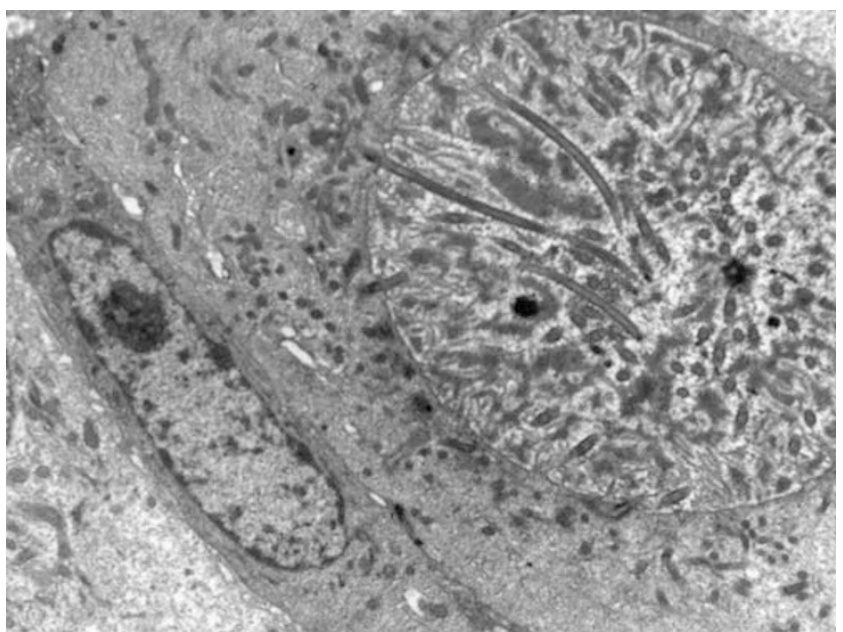

Conclusions: We have presented the ultrastructural findings of 5 patients in which the clinical history was highly suggestive of PCD; however, the derivation of cilia malfunction appears not the result of any recognized primary cause or secondary effect. Instead, the cases presented may illustrate a mechanism by which structurally normal appearing basal bodies and cilia are formed within the respiratory epithelial cells, but, due perhaps to some disruption in a membrane trafficking pathway, not anchored in their normal apical position. This new entity, termed Ciliary Inclusion Disease, represents a novel variant of $\mathrm{PCD}$ and should be considered when performing routine ultrastructural evaluation of ciliary biopsies. 